\title{
Unanticipated risks in morbidly obese patients
}

\author{
Department of Anesthesiology and Pain Medicine, Chung-Ang University Hospital, Seoul, Korea
}

\author{
Young-Cheol Woo
}

In Korea, $32 \%$ of adult are obese. Although this rate is based on Asian body mass index (BMI), obese patients have been increasing suddenly and today anesthesiologists can frequently meet morbidly obese patients (MOPs) in the operating rooms. "Morbid obesity" describes obesity that, if untreated, will significantly shorten the patient's life. The criteria is BMI over $40 \mathrm{~kg} / \mathrm{m}^{2}$ or $100 \%$ over ideal body weight. Studies have reported up to a 12-fold excess mortality rate in association with morbid obesity along with an exceptionally high incidence of co-morbidities [1].

In August and October 2009 issues of Korean Journal of Anesthesiology, two case reports warned the risks in MOPs. Kim et al. experienced a respiratory failure and subsequent cardiac arrest after change to supine position in MOP with a BMI of about $62 \mathrm{~kg} / \mathrm{m}^{2}$ [2]. The patient was bearable in lateral position. As position was changed to supine for urologic procedure under local anesthesia in the operating room, he showed dyspnea and agitation. The anesthesiologists were asked for help but cardiopulmonary resuscitation (CPR) was in vain. And they pointed out that there was no CPR guideline for MOPs. Song et al. reported the patient with dyspnea and chest pain after brachial plexus block (BPB) due to phrenic nerve block whose respiratory reserve was decreased [3]. The patient was morbidly obese and general anesthesia was induced because of the unsuccessful BPB and dyspnea. Although she recovered without any residual complication, this case shows that anesthesiologists should have preoperative consideration for decreased respiratory reserves in MOPs.

These cases give us a lesson that high vigilance is necessary

Corresponding author: Young-Cheol Woo, M.D., Ph.D., Department of Anesthesiology and Pain Medicine, Chung-Ang University Hospital, 224-1, Heukseok-dong, Dongjak-gu, Seoul 156-070, Korea. Tel: 82-2-6299-2584, Fax: 82-2-6299-2585, E-mail: wj12@naver.com

Copyright (C) Korean Society of Anesthesiologists, 2009

(C) This is an open-access article distributed under the terms of the Creative Commons Attribution License, which permits unrestricted use, distribution, and reproduction in any medium, provided the original work is properly cited. for management of MOPs. A very small change such as position change and sedatives administration can make patients lethal. Postoperative analgesics given in general ward may cause respiratory failure in MOPs. Because obesity increases risk of multiple organ failure (MOF) after severe trauma [4], multiple fractures in MOPs may cause perioperative death. MOF is more likely to develop within 72 hours of injury in obese patients than non-obese patients and this probably comes from proinflammatory nature of the obese state. Obstructive sleep apnea syndrome (OSAS) is a predominant cause of sudden death in obese persons. Pickwickian syndrome is the most serious OSAS and the patients go as far as to be found dead [5]. One report shows that $15 \%$ of severely obese men with OSAS died suddenly and unexpectedly due to acute myocardial infarction and pulmonary edema during a 4-year follow-up. Their mean age was 46 years and mean BMI was $41 \mathrm{~kg} / \mathrm{m}^{2}$. They did not have other conventional risk factors [6]. MOPs are at risk for various causes of death.

And also, perioperative complications may develop suddenly and unexpectedly in MOPs, which may cause legal problems. Therefore prudential approach and high vigilance are essential. However it is not easy to make full evaluation in emergency situation. If anesthesiologists are not familiar with MOPs and there is no treatment guideline including CPR yet, the outcomes will be not good. Nowadays, the anesthesiologists are challenged for management of MOPs and should consider the potential risks for caring for the MOPs.

\section{REFERENCES}

1. Drenick EJ, Bale GS, Seltzer F, Johnson DG. Excessive mortality and cause of death in morbidly obese men. JAMA 1980; 243 : 443-5.

2. Kim BS, Lee JH, Kim EJ, Lee SK, Ban JS, Min BW. Cardiac arrest after position change in a morbidly obese patient. Korean J Anesthesiol 2009; 57: 237-41.

3. Song JG, Kim SK, Jeon DG, Kwon MA, Yoo JH. Dyspnea after supraclavicular brachial plexus block in a morbidly obese patient 
due to phrenic nerve block. Korean J Anesthesiol 2009; 57: 511-4.

4. Ciesla DJ, Moore EE, Johnson JL, Burch JM, Cothren CC, Sauaia A. Obesity increases risk of organ failure after severe trauma. J Am Coll Surg 2006; 203: 539-45.

5. Uemura K, Harada K, Shiotani A, Kai A, Urata Y, Yasuhara M, et al. Obesity-sleep apnea (Pickwickian) syndrome: autopsy findings and a medicolegal review. Leg Med 2000; 2: 36-41.

6. Rössner S, Lagerstrand L, Persson HE, Sachs C. The sleep apnoea syndrome in obesity: risk of sudden death. J Intern Med 1991; 230: $135-41$. 